\title{
Socioeconomic status and cardiovascular health in the changing world
}

\author{
Krzysztof S. Gołba, Jolanta Biernat \\ Department of Electrocardiology and Heart Failure, School of Health Sciences, \\ Medical University of Silesia, Katowice, Poland
}

\section{Article p. 487}

Socioeconomic status (SES) is a composite economic and sociological total measure that typically incorporates three indicators: economic status measured as an income, social status measured as an education and work status measured as an occupation. It is commonly believed that SES has a profound association with the health and especially that health outcomes improve as a socioeconomic position improves. Individuals lower in SES have higher rates of morbidity and mortality compared to individuals higher in SES, especially in cardiovascular health outcomes [1].

As early as in the late 1960s Shekelle et al. [2] revealed that the incidence of coronary heart disease (CHD) in middle-aged Caucasian men employed by an industry in metropolitan Chicago was associated with lower educational status. The importance of the type of employment shows a study conducted in the Australian population between 1979 and 1993 - men in manual occupations were at least 35\% more likely to die from CHD than men in professional occupations, and $60 \%$ more likely to die from a stroke [3].

A simple model based on income, education and occupation becomes more complex when one considers conventional coronary disease risk factors. Data from The Oslo Study showed that mortality from CHD and sudden death actually depends on SES assessed using income and educational status but only when coronary risk factors gradients where taken into account [4]. It was confirmed in the Minnesota Heart Survey - improvement in CHD risk over the time may be not related to the education or income because population-wide fac- tors such as higher health knowledge, availability of healthy diet, hypertension treatment, and especially restrictions on cigarette smoking operate beneficially in all SES groups [5].

Recently published data from the research conducted in the Australian population between 1979 and 2006 show remarkable change in the impact of SES gradients on mortality rate for CHD. Differences in mortality rates between lower and higher SES groups narrow for CHD and stroke, indicating that in high-income populations, where fall pattern of cardiovascular mortality exists, the low SES groups benefit more, in absolute terms, than high SES groups [6].

In recent years, particular attention has been paid to women, who are more likely to live in poverty, indicated that gender is a significant covariate acting as an important factor in SES influence on CHD outcomes [7].

Generally, research on relationships between SES and cardiovascular risk factors of patients with CHD was performed in Western Caucasian populations many years ago. SES was found to be strongly associated with the risk of $\mathrm{CHD}$, its prevalence and treatment, as well as late outcomes in the United States and Western European countries. It is not the same in developing countries, where nowadays one can observe the epidemiological transition characterized by the shift from main role of acute infectious diseases to the growing role of chronic diseases, especially different forms of cardiovascular diseases, as a main cause of mortality and morbidity throughout these areas. We are lacking the detailed data because of the under-diagnosis and the low level of treatment of chronic diseases [8].

It is believed that in Central and East European countries similar epidemiological transition

Address for correspondence: Prof. Krzysztof S. Gołba, MD, PhD, FESC, Department of Electrocardiology and Heart Failure, Medical University of Silesia, $7^{\text {th }}$ University Hospital, ul. Ziołowa 45/47, 40-635 Katowice, Poland, tel/fax: +48 3235988 93, e-mail: kgolba@sum.edu.pl

Received: 22.07.2015 Accepted: 25.07.2015 
is in progress and the process is accelerating. The changes in SES of East and Central European population are not homogenous and substantial geographical disparities in health are evident at multiple scales, including these between countries, regions and cities [9]. Data presented by Tubek et al. [10] in this issue of "Cardiology Journal" try to bridge the gap in knowledge concerning Central European countries, especially Polish population and indicate that in Poland the pattern of the impact of SES on cardiovascular outcomes follows the trends in populations of Western countries [10].

Conflict of interest: None declared

\section{References}

1. Marmot MG, Shipley MJ, Rose G. Inequalities in death: Specific explanations of a general pattern? Lancet, 1984; 1: 1003-1006.

2. Shekelle RB, Ostfeld AM, Paul O. Social status and incidence of coronary heart disease. J Chronic Dis, 1969; 22: 381-394.

3. Bennett S. Socioeconomic inequalities in coronary heart disease and stroke mortality among Australian men, 1979-1993. Int J Epidemiol, 1996; 25: 266-275.
4. Holme I, Helgeland A, Hjermann I, Leren P, Lund-Larsen PG. Four-year mortality by some socioeconomic indicators: the Oslo study. J Epidemiol Community Health, 1980; 34: 48-52.

5. Luepker RV, Rosamond WD, Murphy R et al. Socioeconomic status and coronary heart disease risk factor trends. The Minnesota Heart Survey. Circulation, 1993; 88 (5 Part 1): 2172-2179.

6. Page A, Lane A, Taylor R, Dobson A. Trends in socioeconomic in equalities in mortality from ischaemic heart disease and stroke in Australia, 1979-2006. Eur J Prev Cardiol, 2012; 19: 1281-1289.

7. Shaw LJ, Merz CN, Bittner V et al. Importance of socioeconomic status as a predictor of cardiovascular outcome and costs of care in women with suspected myocardial ischemia. Results from the National Institutes of Health, National Heart, Lung and Blood Institute-sponsored Women's Ischemia Syndrome Evaluation (WISE). J Womens Health (Larchmt), 2008; 17: 1081-1092.

8. Chen F, Yang Y, Liu G. Social change and socioeconomic disparities in health over the life course in China: A cohort analysis. Am Sociol Rev, 2010; 75: 126-150.

9. Richardson EA, Pearce J, Mitchell R, Shortt NK, Tunstall H. Have regional inequalities in life expectancy widened within the European Union between 1991 and 2008? Eur J Public Health, 2014; 24: 357-363.

10. Tubek S, Stepkowski M, Szczurowska A et al. Sexual dimorphism in socioeconomic differences regarding the risk factors, symptomatology and management of patients with stable coronary artery disease in Poland. Cardiol J, 2015; 22: 487-494. 